\title{
Nanorobots and their Biomedical Applications
}

\author{
T. Gayathri \\ Department of Biomedical \\ Engineering \\ KPR Institute of Engineering and \\ Technology \\ Coimbatore, India
}

\author{
S. Sree Sanjanaa Bose \\ Department of Biomedical \\ Engineering \\ KPR Institute of Engineering and \\ Technology \\ Coimbatore, India
}

\author{
T. Poornima \\ Department of Electrical and \\ Electronics Engineering, \\ Sri Shakthi Institute of Engineering \\ and Technology, \\ Coimbatore, India
}

\begin{abstract}
The application of nanotechnology with the new electronic materials led to the development of nanorobots. Nanorobots, in the nano scale region are capable of entering the cell for diagnosis, treatment and surgery. Due to its wide range of applications, nanorobots are designed with specific materials and design technologies. Since the biocompatible materials are used in the design of nanorobots, they are chemically inert and the problem of toxicity is avoided. The nano scale size allows the targeted delivery of drugs to the specific site without affected the normal surrounding cells. Nanorobots can move freely in the bloodstream due to the Brownian motion. This review clearly explains the various biomedical applications of the nanorobots.
\end{abstract}

Keywords—Nanorobots, diagnosis, treatment, surgery

\section{INTRODUCTION}

Nanorobotics is an emerging field of nanotechnology which deals with design and construction of devices at an atomic, molecular or cellular level. These hypothetical nanorobots will be extremely small and would transverse inside the human blood. As these nanorobots would have special sensors to detect the target molecules, it can be programmed to diagnosis and treat various vital diseases [1]. Nanorobots would constitute any "smart" structure capable of actuation, sensing, signaling, information processing, intelligence, manipulation and swarm behavior at nano scale $\left(10^{-9} \mathrm{~m}\right)$. Nanomedicine offers the prospect of powerful new tools for the treatment of human diseases and the improvement of human biological systems. Nanomedicine is the process of diagnosing, treating, and preventing disease and traumatic injury, of relieving pain, and of preserving and improving human health, using molecular tools and molecular knowledge of the human body [2].

A nanorobot is an extremely small robot designed to perform a specific task with precision at nano-scale. They are also known as nanorobots or nanoids. The design of nanorobot is derived from biological models of bacteria. Carbon will likely be the principle element used in construction of nanorobot and comprised probably in the form of diamond/ diamondoid (includes pure diamond and crystalline allotrope of carbon) or fullerene nanocomposites. A nanorobot can be made of mechanical parts such as bearing, gears, motors etc. The outer shell of nanorobot is likely to be constructed using diamondoid material due to their inert properties, high thermal conductivity and strength. The super-smooth surfaces could reduce the chances of triggering the body's immune system. The nanoscale gears and other components designed for special purposes could be constructed using elements like hydrogen, sulfur, oxygen, nitrogen, silicon etc [3].
The ability to manufacture nanorobots may result from current trends and new methodologies in fabrication, computation, transducers and manipulation. CMOS VLSI design using deep ultraviolet lithography provides high precision and a commercial way for manufacturing early nanodevices and nanoelectronics systems [4]. To validate designs and to achieve a successful implementation, the use of VHDL (Verification Hardware Description Language) has become the most common methodology utilized in the integrated circuit (IC) manufacturing industry [5]. CMOS with submicron SoC design could be used for extremely low power consumption with nanorobots communicating collectively for longer distances through acoustic sensors [6].

This review focuses on the various applications of nanorobots in the field of surgery, neurology, dentistry, hematology, microbiology, cancer treatment and gene therapy.

\section{BIOMEDICAL APPLICATIONS OF NANOROBOTS}

\section{A. Nanorobots in Surgery}

In the traditional surgery methods, the surgeons will cut the healthy tissues and expose the organs or parts to be operated. Due to the advances in the medical technology, minimally invasive surgical methods are being used widely. The advantage of minimally invasive surgery includes less scarring, less pain, reduced infection rates and faster rehabilitation. With the miniaturized size, nanorobots can easily enter the body cavities. After the entry into the cells, nanorobots can be controlled by the surgeon using a computer. With the advent in the nanotechnology, many tools are produced on the nanoscale to perform surgery, even on a single cell. Surgical blades coated with nanoparticles, nanoneedles, nanolasers, nanocoated catheters, nanocontoured implant surfaces are the applications of nanotechnology in the surgery [7].

Atomic Force Microscopy (AFM) can scan the images in the nanoscale and finds application in the imaging and therapeutics. AFM tips are approximately $20 \mathrm{~nm}$ or less and can produce high resolution images. These AFM tips can perform local surgery within the cellular level. The advantage of AFM robotics is that they can image as well as manipulate the samples in nano scale. AFM based nanorobots uses three techniques to rectify the limitations of conventional AFM. Augmented reality interface uses a joystick for the positioning and motion control. The joystick receives three-dimensional interaction forces which can be controlled easily. AFM tips can be functionalized with various ligands and antibodies to 
study the specific molecular interactions. Specific reagents attached to the AFM tips allow the local in vitro local drug delivery. The surface changes sensed by the AFM tips provide real-time online monitoring system by the visual feedback of living samples [8].

\section{B. Nanorobots in Neurology}

Nanorobots in the field of neurology, has been an active area of practical research and development. The innovation in nanotechnology and introduction of nanorobots can be helpful in neurosurgery for various applications like improving the detection of pathology, minimally invasive intracranial monitoring and pharmaceutical delivery and many others. The spinal cord and nerve injury is a serious complication in the field of neurosurgery. The major difficulty faced is reconnection of transected nerves and promoting the regeneration of axons through enriched scaffolds. The restoration function can be activated only by reconnecting the damaged nerves. Performing the surgery at that minute scale is the limitation faced by the physicians. The manipulation of individual axons is made possible with the help of nanosized devices. A nano knife of size $40 \mathrm{~nm}$ in diameter is effective in axon surgery. The motion of axons in surgical field is controlled by manipulating the polarizable objects in electric field, by the process called dielectrophoresis. Following this the two ends of the nerves are connected by electrofusion or laser induced cell fusion [9].

Brain aneurysm is the formation of clot in the blood vessels that supply oxygen to the brain. This life threatening bleeding intracranial disease due to the leakage or rupture of the aneurysm is found mostly between the base of the skull and underneath of the brain. The nanotechnology has helped in the development of robots of nano-sized for prognosis of brain aneurysms. The three key concepts of the construction of nanorobots are equipment prototyping, manufacturing approach and inside-body interaction. The prototyping of medical nanorobots requires the equipment prototyping which is established with computational nanotechnology which drives as a key tool for the rapid and effective development of instrumentation of robot. The manufacturing approach focuses on creating a biochip integrated robot with the help of biomaterials, photonics and nano bioelectronics. The insidebody interaction deals with the compatibility of the nanorobots to gel and interact with the biological parts and chemical reactions. The morphology of the nanorobots is derived from the cell morphology, proteomics and microbiology. The nanorobots designed for the prognosis of brain aneurysm should be efficient to detect the endothelial injury by tracking along the blood vessels before the subarachnoid haemorrhage develops. The chemical nano biosensor helps the nanorobots in this process of detection because the tiny sized biomolecules are difficult to be detected reliably. Nitric oxide synthase (NOS) level in the brain helps in detecting the presence of intracranial aneurysm. The detection thresholds are set based on the protein expression and if the NOS level is found in minute, a signal of $50 \mathrm{nA}$ is send. If the level of NOS exceeds the threshold like 100 proteomic signal transduction it is a sign of strong evidence for the presence of intercranial aneurysm. Following this, the nanorobots send the information of the location of the vessel and the dimension of the bulb [10].

\section{Nanorobots in Dentistry}

Nano dentistry has become an emerging field with the specialized use of nanorobots at a significant routine. The applications of nanorobots in the field of dentistry are cleaning, cosmetics for teeth whitening, root canal treatment, treatment of hypersensitivity, alignment of the teeth, improving the durability of the teeth, and oral analgesia. The various tissue engineering techniques are incorporated for the complex tooth repair. The oral analgesia is established by orally administering a mixture comprising millions of nanorobots which have the ability to penetrate into gingival sulcus deep down the pulp. The nanorobots in a controlled manner provide the medicine at the specific areas of treatment. Root canal treatments and treatment of any dental infection is made possible with the ability of the nanorobots to be enveloped within a capsule for interaction and detection. In the process of root canal treatment the use of tiny camera inside the nanorobots for the visualization of infected root aiding the doctor in the procedure makes the treatment a successful one. In 2011, the success rate of the root canal treatment is $70 \%$ as reported National Health Service, which promises for improvement in this area. Biologically, autologous whole tooth replacement with nanostructured composite has completely revolutionised dentition replacement therapy. Sapphire, a covalently bonded artificial material having 200 times the hardness strength than ceramic and standard whitening sealant has replaced enamel in the artificial tooth. Nanocomposite is manufactured by nanoparticles distributed in resins. The nano filler is a combination of aluminium and silicon powder with refractive index of 1.503. It blends well with natural tooth structure compared to the conventional composites. The role of nanorobots in the treatment of dentine hypersensitivity has increased attraction. The number of dentinal tubules in hypersensitive teeth is higher compared to the normal teeth. Nanorobots perform the selective ablation when penetrated into the dentinal tubules which prevents the stimuli from inducing the pain response. Nanorobots also help in dental care by the incorporation of nanorobots into the mouthwash and toothpaste which when used at a routine basis directly manipulates the periodontal tissues aiding the process of tooth repositioning [9].

\section{Nanorobots in Hematology}

The applications of nanorobotics and nanomedicine in the field of hematology are at the research level and the applications are under study. The applications of nanorobots in hematology range from transfusions of non-blood oxygen carrying to restoring primary hemostasis. Nanorobot dubbed respirocyte is under design for aiding the process of blood transfusion. The size of the robot would be 1000 nanometers with its components constructed at nano level. It will comprise a onboard computer with a diameter of $58 \mathrm{~nm}$, oxygen and carbon dioxide loading rotors with a diameter of $14 \mathrm{~nm}$. The three main functions of nanorobot dubbed respirocyte while travelling through the bloodstream are collection of oxygen from the respiratory system, collection of carbon dioxide from tissues and metabolizing the circulating glucose to power its own functions. This robot is equipped to carry 236 times more oxygen per unit volume than the red blood cells. 
The hemostasis is second application where nanorobotics is focused for research. The hemostasis involves a range of steps which requires various promoters and inhibitors balancing thrombosis and fibrinolysis. The hemostasis when works perfectly, halts the process of bleeding and improves the reparing process of infected blood vessel. The challenge faced in physiologic hemostasis is the average bleeding time is nearly 5 minutes. Nanorobotics can be used in solving this problem. During the platelet transfusion, the patients may develop infection with pathogens and leading to development of an immune response. The artificial mechanical platelet, "clottocyte" nanorobot has been proposed for imitating this function. The size of the proposed robot is two micron along with a mesh of $0.8 \mathrm{~nm}$, incorporated with hemostasis promoting protein which will be used for binding the vessel injury during hemostasis.

The third potential application of nanorobots in the field of hematology is the role as phagocytic agents. Nanorobots proposed are named as "Microbivores". Microbivore, an artificial mechanical phagocyte designed by Robert A. Freitas, is a nanorobotic device that safely eradicates the blood-borne pathogens. It is an oblate spheroidal nanomedical device comprising of 610 billion precisely arranged structural atoms plus 150 billion gas molecules. The nanorobot has numerous customizable binding sites on its external surface for antigens and pathogens. It is believed to have the potential of curing septicemia within hours of administration and is more effective nearly 80 times better than the natural phagocytic capabilities. The realization of these nanorobotics applications can be helpful in treatment of infections [10].

\section{E. Nanorobots in Microbiology}

Microbiology has been a promising platform for realizing the feasibility of the robotic applications at nano level. Inspite of the successful construction of nanorobots with effective functionality, their performance within the vascular system is limited due to the difficulties faced at transportation and propulsion stage. The propulsion of nanorobots can be established by the effective coupling of these robots with the magnetobacteria such as magnetococcus, magnetospirillum magnetotacticum and magnetospirillum magneticum. The complex part of the nanorobots integrated into the magnetotactic bacterium is the bacterial cell component. Marine Magnetotactic spirillum is the smallest magenetotactic bacteria of size $500 \mathrm{~nm}$ but due to its limitation in its speed, magnetotactic cocci are preferred for intravascular function. The magnetic field plays the role of guiding the magnetotactic bacteria. The components that are responsive to magnetic field are called Magnetosomes. They are prokaryotic pseudoorganelles consisting of 20 magnetite crystals, of size $50 \mathrm{~nm}$ in diameter enclosed in a prokaryotic cell membrane. Magnetite crystals are composed of Ferrous oxide. Magnetotactic cocci are found to have a predictable pattern and consistency in following the geomagnetic lines. A highly customizable structure can integrated with the bacteria, carrying artificial antibodies and medicine for delivering at the target site. They also function as sensors and collect information about the infected site. Larger robots have the potential to move through large vessels while smaller robots can work well at capillaries but the control of velocities cannot be achieved. The comprising two-component robotic system has been proposed where larger system controls the transport through larger vessels and the smaller system focuses on delivering smaller medications at smaller vessels and capillaries [11].

\section{F. Nanorobots in Cancer Treatment}

With the advancement of technologies in the medical field, the treatment of cancer can be successfully established. The early diagnosis of the cancer has been the factor that decides the chances of survival of a cancer patient. It is highly important to detect the cancer before metastasis stage for the successful treatment of cancer. Nanorobots can be integrated with chemical biosensors for detecting the presence of tumour at an early stage. E-Cadherin is a tumour suppressor protein belonging to a class of transmembrane proteins. The Cadherin-1 also known as CAM 120/80 is a cell adhesion molecule and is dependent on calcium ions to function. The intensity of e-cadherin can be measured by the nanorobots for detecting the presence of tumour at the early stage. The nanorobots can be introduced in tumour resection surgeries. A radioactive colloid injection will be administered to the patient into the prostate a day prior to the resection surgery and radioisotope guided lymph node dissection will be performed which is more quick in detecting the metastasis compared to open lymph dissection. This procedure can be improved by the incorporation of nanorobots eliminating the patient admission prior to the surgery [9].

An important aspect of cancer treatment is the targeted drug delivery in order to reduce the side effects of popular cancer therapy, chemotherapy. As discussed earlier, the nanorobots have the ability to travel within smaller vessels and deliver medication at target sites. The nanorobots are highly potential to be used in cancer therapy. The conventional chemotherapy has the limitation in dosage administration because it affects the normal cells along with the tumour cells. The limitation can be overcome by nanotechnology with the selectivity of treatment sites. Nanorobots can autonomously detect the cancer cells and delivers the drug at the site of cancerous areas. The nanorobot can be constructed to selectively respond to different cell surface receptors and the level of payload to be administered can also be controlled. The engineered DNA strand is used for the construction of this type of nanorobot that has a desired tertiary structure. The nanorobot upon binding with the desired target, unfolds its structure to administer the dose at the cancerous cells. Another nanorobot that has the potential to travel along the blood vessel and release the medication at the target area is built of synthetic elements, called Pharmacyte. This nanorobot contains therapeutic payload having self sufficient energy generation and locomotive function to move across the tissues and cell membranes [10].

\section{G. Nanorobots in Gene Therapy}

Nanorobots play a significant role in treatment of gene related diseases. The medical nanorobots have the ability to cure the genetic diseases by comparing the molecular structure of DNA and the protein sequences. The abnormality or irregularity can be treated by the modifying the genetic structure at the nano level. It is proved by experiments that cell repair can be replaced by chromosomal replacement therapy due to its high efficiency. An assembler built repair 
vessel is placed inside nucleus of a human cell which performs the genetic maintenance. The molecular structure of DNA and protein sequences are compared with the information stored in the database of the Nano computer. If any abnormality is found, the unfolded strand will be corrected by attaching the proteins to the DNA chain and then it regains the original position. The size of the repair vessel will be of $50 \mathrm{~nm}$ capable for therapies and cures the cancer, viral infections and arteriosclerosis [11].

\section{CONCLUSION}

The nanorobots which revolutionize the medical field, helps in the diagnosis and treatment of many diseases including cancer. Nanorobots are site-specific, non-invasive, computer control of delivery and reduce the side effects caused by the conventional therapy techniques. This leads to the early diagnosis of diseases and improved therapy at low cost. The usage of nanorobots for the conventional diagnosis and treatment is possible in near future.

\section{ACKNOWLEDGMENT}

The authors would like to thank the management of KPR Institute of Engineering and Technology, Coimbatore for their support and encouragement in publishing this manuscript.

\section{REFERENCES}

[1] M. Apoorva, and V. Kishore, "The Promising Future in Medicine: Nano robots", Sci. Educ., vol. 2(2), pp. 42-47, 2014.

[2] P. Khulbe, "Nanorobots: a review", Int. J. Pharm. Sci. Res., vol. 5(6), p. 2164,2014

[3] S. Saxena, B.J. Pramod, B.C. Dayananda, and K. Nagaraju, "Design, architecture and application of nanorobotics in oncology", Indian J. Cancer, vol. 52(2), pp. 236-241, 2015.

[4] A. Cavalcanti, B. Shirinzadeh, M. Zhang, and L.C. Kretly, "Nanorobot hardware architecture for medical defense", Sensors, vol. 8(5), pp. 2932-2958, 2008.

[5] S.M.M.R. Al-Arif, N. Quader, A.M. Shaon, and K.K. Islam, "Sensor based autonomous medical nanorobots: A cure to demyelination", J. Sel. Areas Nanotechnol., pp.10-15, 2011.

[6] A. Cavalcanti, B. Shirinzadeh, R.A. Freitas Jr, and T. Hogg, "Nanorobot architecture for medical target identification", Nanotechnology, vol. 19(1), p.015103, 2007.

[7] S. Mali, "Nanotechnology for surgeons", Indian J. Surg., vol. 75(6), pp. 485-492, 2013.

[8] B. Song, R. Yang, N. Xi, K.C. Patterson, C. Qu, and K.W.C. Lai, "Cellular-level surgery using nano robots", J. Lab. Autom., vol. 17(6), pp. 425-434, 2012.

[9] Y. Saadeh, and D. Vyas, "Nanorobotic applications in medicine: current proposals and designs", Am. J. Robotic Surg., vol. 1(1), pp. 4 $11,2014$.

[10] J. Gupta, "Nanotechnology applications in medicine and dentistry", J. Investig. Clin. Dent., vol. 2(2), pp. 81-88, 2011.

[11] M. Li, N. Xi, Y. Wang, and L. Liu, "Progress in nanorobotics for advancing biomedicine”, IEEE Trans. Biomed. Eng., pp. 1-18, 2020. 